\title{
Análisis de cerámicas romanas Terra Sigillata mediante espectroscopía de plasmas inducidos por láser (LIPS)
}

\author{
A.J. LÓPEZ, G. NICOLÁS, M.P. MATEO, V. PIÑÓN, A. RAMIL, A. YÁÑEZ \\ Universidade da Coruña, Laboratorio de Aplicacións Industriais do Láser, Mendizábal s/n, 15403 Ferrol (A Coruña), España \\ E-mail adress: ajlopez@cdf.udc.es
}

\begin{abstract}
En este trabajo se ha aplicado la espectroscopía de plasmas inducidos por láser (LIPS) al análisis elemental de cerámicas romanas Terra Sigillata procedentes de distintos centros de producción. Para cada muestra se han obtenido los espectros característicos tanto del engobe como de la pasta y los perfiles de composición en profundidad. En todos los casos analizados, los elementos Fe y Ca son los que marcan la transición entre el engobe y la pasta en estas cerámicas. El gradiente de composición de otros elementos tales como el Si o el Al presenta diferencias entre las distintas piezas. Se han realizado también análisis complementarios por microscopía electrónica de barrido con espectroscopía de rayos X (SEM - EDX) para determinar las diferencias morfológicas y obtener una verificación de la composición química.
\end{abstract}

Palabras clave: LIPS, Terra Sigillata, perfil de composición, cerámicas arqueológicas

Analysis of roman pottery Terra Sigillata by laser induced plasma spectroscopy (LIPS)

Laser induced plasma spectroscopy (LIPS) has been applied for the elemental analysis of roman pottery Terra Sigillata from different ceramic production centers. For each sample, representative LIP spectra of slip and body were obtained in addition of compositional depth profiles. In all the cases investigated, calcium and iron are the elements which best define the transition between slip and body in these ceramic samples. Compositional gradient of other elements like silicon and aluminum depends on the samples. Moreover, complementary scanning electron microscopy coupled with energy dispersive X-ray spectroscopy (SEM - EDX) examinations were carried out to analyze morphological differences and to obtain verification of chemical results.

Keywords: LIPS, Terra Sigillata, compositional depth profile, archaeological ceramics

\section{INTRODUCCIÓN}

La Terra Sigillata es una cerámica fina de mesa típica de la Época Romana que se caracteriza por estar dotada de un engobe sinterizado que asemeja la apariencia de un barniz de tonos rojizos. La producción de Terra Sigillata se inició en Italia Central en el siglo I a.C. y desde allí se extendió por todo el Imperio, siendo popular hasta los siglos IV-V d.C. Sus restos son de gran interés arqueológico por las implicaciones económico-comerciales, puesto que se trata de objetos de extensivo comercio en el Imperio Romano [1], sirviendo además como indicadores de la capacidad artística y tecnológica desarrollada en la época. El examen científico de estas cerámicas puede aportar información valiosa a la investigación arqueológica tanto en la asignación de procedencias como en el redescubrimiento de las tecnologías de producción [2,3]. Con este propósito se han venido utilizando distintas técnicas de microscopía (óptica y electrónica) y espectroscopías de tipo electrónico, atómico, molecular, nuclear o de rayos-X; pudiendo establecerse una clasificación de las mismas en dos grandes grupos: aquellas técnicas que pueden aportar información cualitativa o cuantitativa de microdominios homogéneos en cada pieza [4-14] y las utilizadas para el análisis cuantitativo de series de muestras, como método para establecer agrupamientos e identificar procedencias $[9,10,15-20]$. En general, todas ellas requieren la preparación de muestras (fragmentación, pulverización, o disolución) y sólo pueden llevarse a cabo como métodos de laboratorio. La búsqueda de técnicas alternativas que no requieran preparación de la pieza y que, de forma rápida y con un moderado gasto y esfuerzo, permitan analizar un gran número de muestras, nos ha llevado a considerar el potencial analítico de la espectroscopía de plasmas inducidos por láser (LIPS) que, en los últimos años, se ha venido aplicando de forma creciente en el análisis de materiales arqueológicos, tanto metales como cerámicas o vidrios [21-26].

En la técnica LIPS, un haz láser pulsado se hace incidir sobre la superficie de una muestra. Si la energía depositada supera un cierto umbral da lugar a la formación de un plasma sobre el área irradiada por el haz. La detección y el análisis del espectro de emisión de este plasma proporcionan información detallada acerca de la composición elemental de la superficie alcanzada en cada pulso. Dado que el haz láser puede enfocarse de manera que su diámetro se encuentre, típicamente, en el rango micrométrico, esta técnica permite la caracterización elemental de la superficie con una gran resolución espacial mediante un barrido del láser sobre la superficie. Además, haciendo incidir repetidamente el haz sobre un mismo punto de la muestra pueden realizarse análisis capa a capa y obtener 
perfiles de composición en profundidad [27].

Aunque existen otras técnicas de análisis con mayores niveles de sensibilidad para los distintos elementos, LIPS presenta importantes ventajas en el estudio de materiales de interés cultural o arqueológico. En primer lugar, se trata de una técnica cuasi no destructiva puesto que no requiere preparación especial de la muestra y la cantidad de material necesario para el análisis es muy pequeña, del orden o incluso menor de $1 \mu \mathrm{g}$. Por otra parte los análisis LIPS no requieren condiciones ambientales especiales, pudiendo realizarse a presión atmosférica y temperatura ambiente. Todo ello, unido a su capacidad para analizar directamente muestras no conductoras tales como cerámicas antiguas y realizar análisis in situ, aumenta sus ventajas frente a otras técnicas convencionales [21]. Entre sus limitaciones cabría destacar la dificultad de realizar análisis cuantitativo debido a la ausencia de patrones y a la importancia de los efectos de matriz, además de una baja sensibilidad en ciertos casos.

Una aplicación particularmente interesante de LIPS se encuentra en el análisis de cerámicas con diferentes capas de decoración, esmaltes o barnices [23,25], en particular cuando se pretende obtener perfiles de composición en profundidad para distinguir entre las diferentes capas o entre el fino barniz y el cuerpo de la cerámica. En este trabajo, la técnica láser se utiliza como herramienta para distinguir las diferencias en composición elemental entre el engobe y la pasta de los fragmentos de Terra Sigillata. Los resultados obtenidos muestran la capacidad de la técnica LIPS para llevar a cabo este propósito. Además, este análisis elemental cualitativo realizado por LIPS, se compara con los datos obtenidos mediante SEM-EDX.

\section{EXPERIMENTAL}

El dispositivo experimental utilizado para los análisis LIPS se muestra de manera esquemática en la Figura 1. Un haz láser pulsado se enfoca sobre la pieza cerámica dando lugar a la formación de un plasma cuya luz se recoge en el espectrógrafo equipado con una cámara ICCD (Intensified Charge Coupled Device) que permite obtener su espectro de emisión. Una caja de conexiones I/O Box (Input/Output Box), permite la

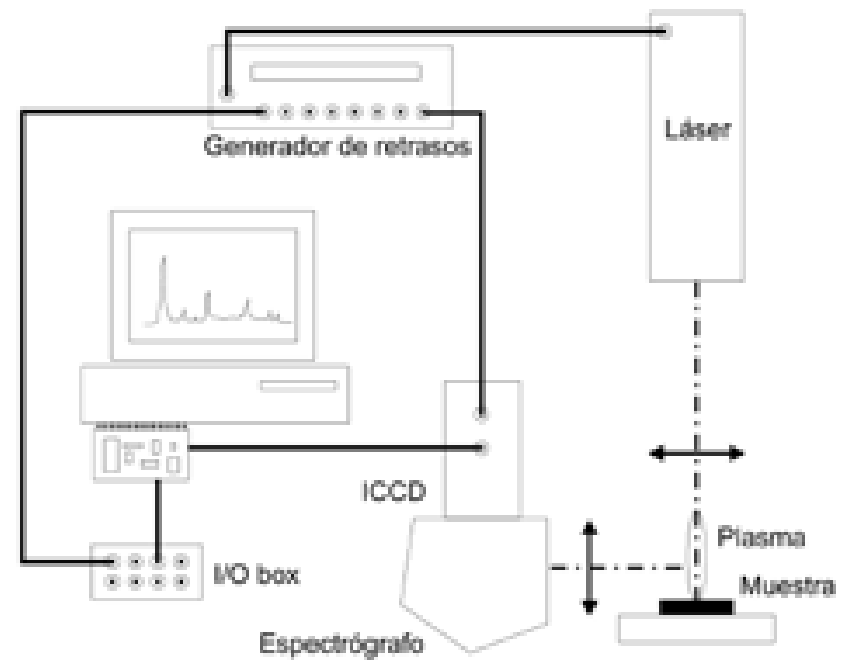

Fig. 1- Esquema del dispositivo experimental empleado para los análisis LIPS.

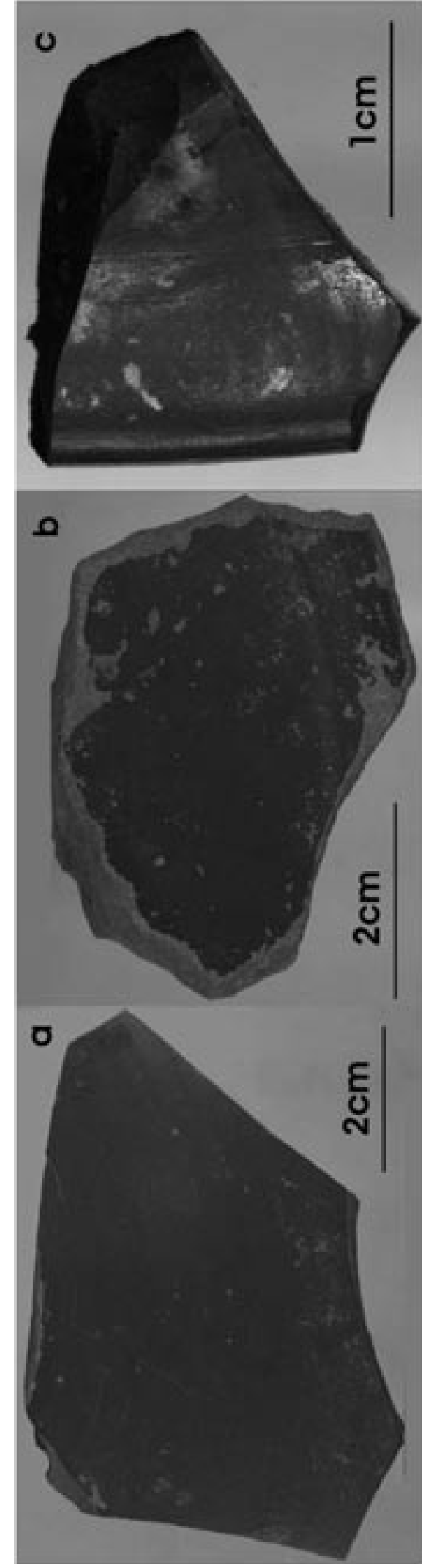

Fig. 2- Fotografías de las muestras de Terra Sigillata analizadas: (a) Tricio, (b) Andújar y (c) La Graufesenque. 
conexión entre el ordenador y la fuente láser con el fin de sincronizar esta con la adquisición de datos. Para minimizar el tamaño del spot y los efectos térmicos de la radiación sobre la pieza analizada, se utilizó como fuente de excitación el tercer armónico de un láser pulsado de Nd:YAG (longitud de onda de $355 \mathrm{~nm}$ ) con una frecuencia de repetición de 10 $\mathrm{Hz}$ y con una anchura de pulso de 6 ns (FWHM), siendo el área irradiada de unos $0.5 \mathrm{~mm}^{2}$ hasta $2 \mathrm{~mm}^{2}$. El espectrógrafo, con una red de difracción de 1200 líneas/mm, recibe la radiación del plasma mediante una lente y su salida se recoge en el detector ICCD con un rango espectral de $180 \mathrm{~nm}$ a 850 $\mathrm{nm}$. La apertura de la cámara ICCD se controla mediante el generador de retrasos que se dispara mediante una señal TTL (Transistor-Transistor Logic) proporcionada por el láser. La ICCD adquiere la señal del plasma durante 500 ns con un retraso de 0 ns a $500 \mathrm{~ns}$ respecto del disparo del láser, con el fin de favorecer la observación de las líneas de emisión iónicas y las líneas de emisión atómicas, respectivamente [28]. Una descripción más detallada del montaje experimental puede encontrarse en [29].

Sobre la base de la información arqueológica suministrada, las piezas seleccionadas para análisis, que se muestran en la Figura 2, fueron asignadas a alfares de los dos más importantes centros de producción en la Península Ibérica, Tricio (Logroño) y Andújar (Jaén) y a los talleres gálicos de La Graufesenque (Milau, Aveyron). Todas las piezas corresponden a la época altoimperial (ss. I y II d.C). Debido a su forma y tamaño irregulares, se preparó un útil de sujeción y se utilizó un sistema óptico basado en láseres de He-Ne para posicionarlas adecuadamente con respecto al sistema de adquisición. Todos los análisis se llevaron a cabo al aire, a temperatura ambiente y presión atmosférica.

\section{RESULTADOS Y DISCUSIÓN}

En una primera etapa se seleccionó la ventana espectral en el rango de $260 \mathrm{~nm}$ a $600 \mathrm{~nm}$ para el análisis LIPS teniendo en cuenta la composición característica de las arcillas estudiadas. Las principales líneas de emisión de los elementos que componen las piezas se encuentran en el rango de $260 \mathrm{~nm}$ a $340 \mathrm{~nm}$. La asignación de las líneas de emisión en el espectro se realizó mediante un programa desarrollado en nuestro laboratorio [30] y utilizando la base de datos del NIST [31].

A continuación se llevaron a cabo varios experimentos para seleccionar los parámetros de trabajo óptimos, teniendo en cuenta el tipo y morfología del material que se iba a analizar. La densidad de energía, el número de acumulaciones y otros parámetros relacionados con el dispositivo de detección se ajustaron para obtener una alta relación señal-ruido de las líneas atómicas y una buena reproducibilidad. Por otra parte, con el fin de poner de manifiesto la transición engobe-pasta se utilizaron unas condiciones de irradiación "suaves" con una densidad de energía de, aproximadamente, $2.0 \mathrm{~J} / \mathrm{cm}^{2}$; como compromiso entre la señal espectral y la resolución en profundidad. En estas condiciones se consigue además preservar el objeto arqueológico de un daño significativo.

Cada muestra se analizó en diferentes puntos del engobe de ambas caras y también en una superficie de fractura fresca para obtener el espectro característico de la pasta. En la Figura 3 se muestran dos espectros representativos del engobe $y$ la pasta de la muestra de Tricio. Con el fin de aumentar su intensidad, los espectros se han obtenido acumulando la señal debida a 3 pulsos láser en el caso del engobe y 5 pulsos en
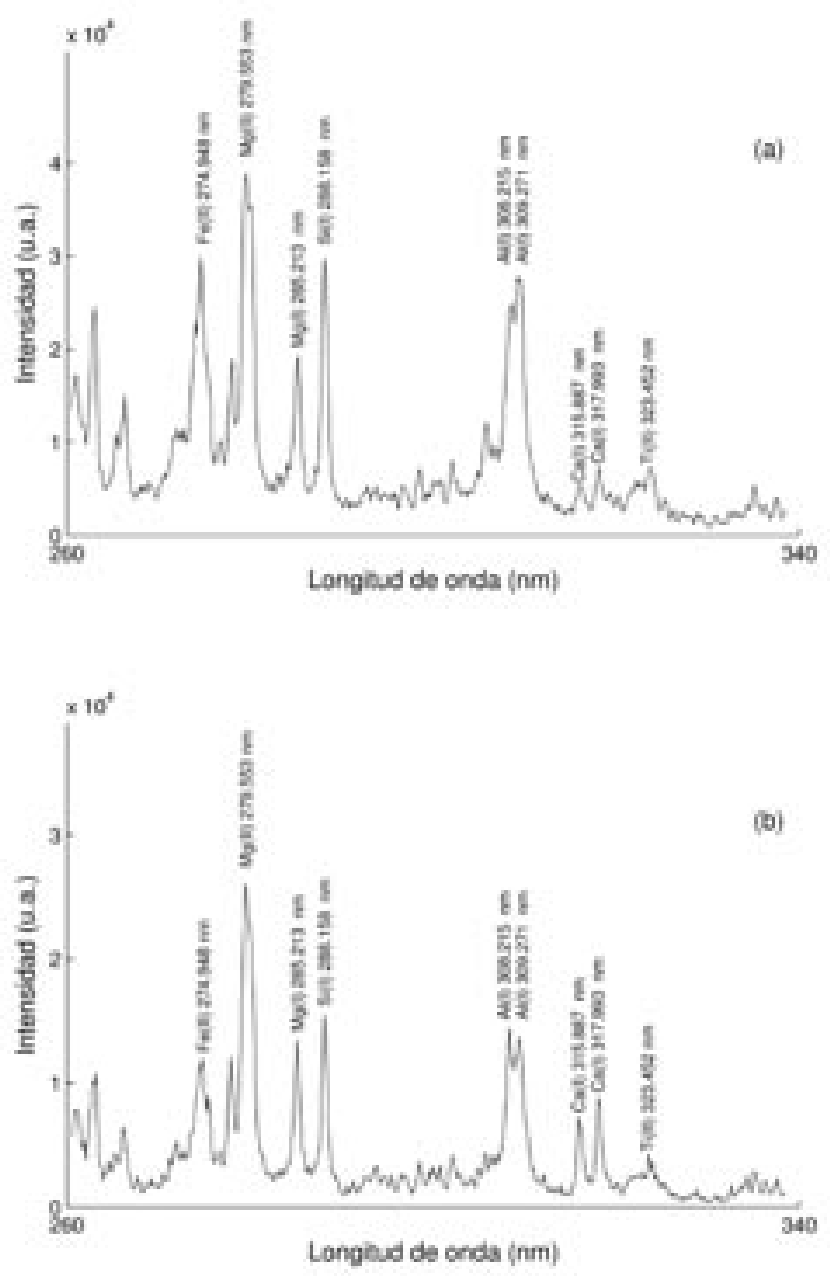

Fig. 3- Espectros LIPS característicos de (a) el engobe y (b) la pasta de la muestra de Tricio, en la región de $260 \mathrm{~mm}$ a $340 \mathrm{~mm}$, obtenidos mediante irradiación láser de $355 \mathrm{~nm}$ y acumulando 3 y 5 pulsos láser, respectivamente.

el caso de la pasta. Como puede observarse, se detectaron los mismos elementos $\mathrm{Fe}, \mathrm{Mg}$, Si, $\mathrm{Al}$, Ca y Ti en ambas zonas de la muestra, pudiéndose apreciar sin embargo pequeñas diferencias relativas entre las intensidades de los distintos elementos, indicando cambios en su proporción. Conviene tener en cuenta que los resultados obtenidos son cualitativos dado que por ahora no se dispone de patrones para estos materiales arqueológicos. Para corroborar el análisis realizado por LIPS, tanto el engobe como la pasta se analizaron mediante SEM-EDX.

Las micrografías obtenidas por SEM, que se muestran en la Figura 4, se realizaron en secciones correspondientes a fracturas frescas utilizando electrones secundarios. En ellas pueden apreciarse claramente las diferencias morfológicas entre engobe y pasta. El engobe de unas $25 \mu \mathrm{m}$ de espesor y con un alto grado de sinterización, puede distinguirse claramente de la pasta, más porosa.

Los análisis EDX se realizaron en, al menos, cinco áreas diferentes de cada muestra, tanto en el engobe, como en la pasta. La Tabla 1 muestra el porcentaje en peso de los elementos detectados y las correspondientes desviaciones típicas. Como era de esperar los resultados están en buen acuerdo con los obtenidos mediante LIPS, aunque algunos aspectos deben comentarse en detalle. Los constituyentes mayores de las cerámicas, $\mathrm{Si}, \mathrm{Al}$ y $\mathrm{O}$, así como otros elementos tales como K, 

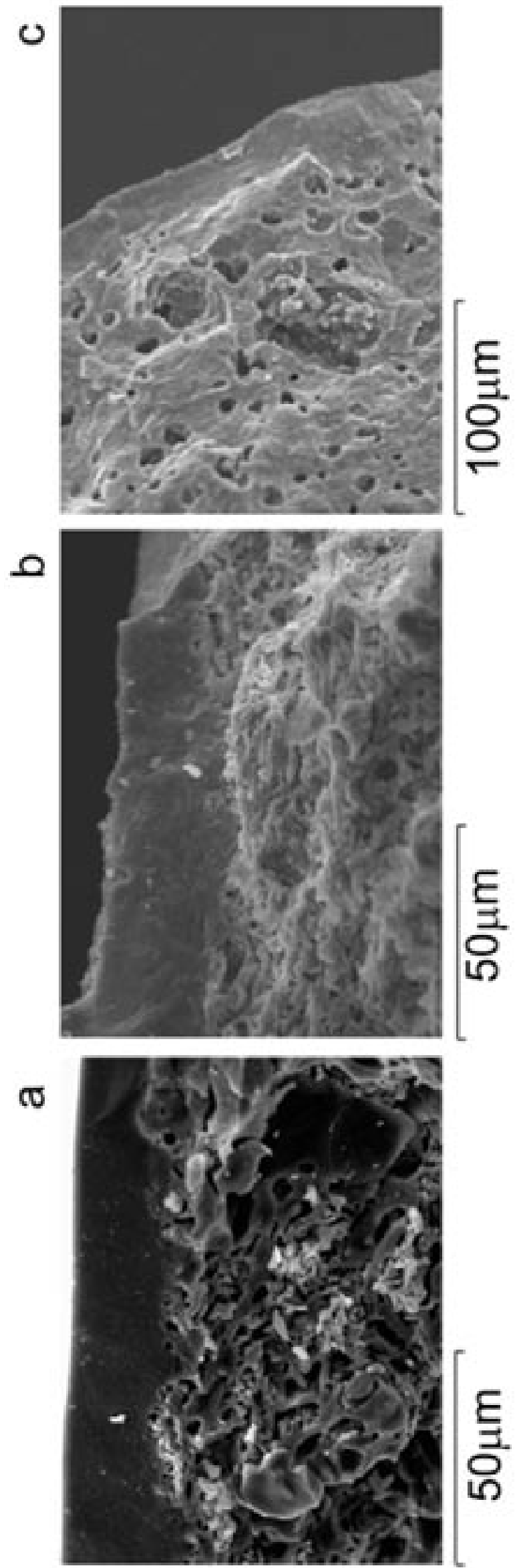

Fig. 4- Imágenes SEM de la fractura fresca de muestras de Terra Sigillata: (a) Tricio, (b) Andújar y (c) La Graufesenque.
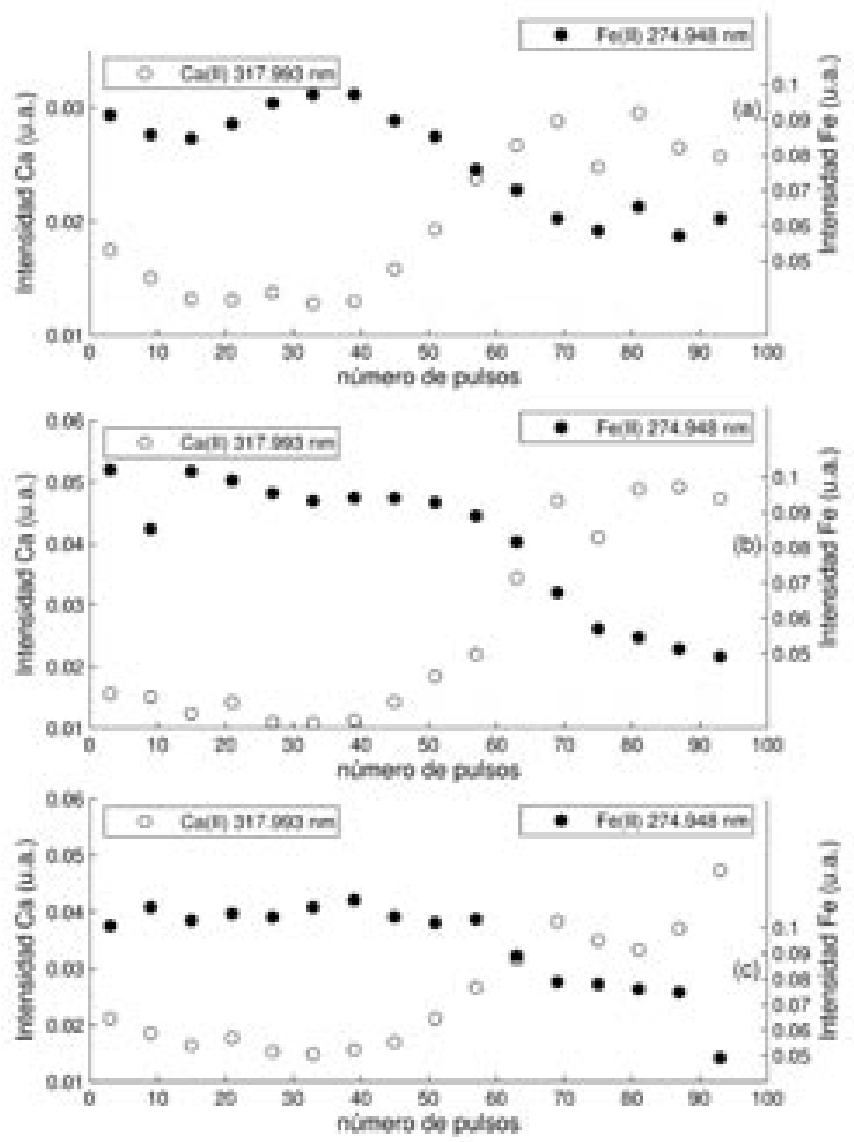

Fig. 5- Perfiles en profundidad de Fe (II) $274.948 \mathrm{~nm}$ y Ca(II) 317.993 nm de las muestras: (a) Tricio, (b) Andújar y (c) La Graufesenque, en función del número de pulsos (i.e. profundidad creciente). Los valores de intensidad se han obtenido acumulando 6 pulsos láser.

Ca y Fe aparecen en los espectros EDX, mientras que Mg y Ti, que pueden apreciarse claramente en los espectros LIPS, no fueron detectados mediante EDX en el engobe de las muestras de Tricio y Andújar. Debe hacerse notar también que las líneas de emisión más intensas del K están fuera del rango espectral considerado en el presente estudio LIPS y las líneas del O se ven enmascaradas por las intensas líneas de los metales.

Mediante la ablación repetida de la superficie de la muestra en el mismo punto de irradiación se ha llevado a cabo el análisis de composición en profundidad. El perfil obtenido ofrece la oportunidad de investigar la distribución elemental desde el engobe hasta la pasta. Hay que señalar que el procedimiento no es directo debido a que la señal de intensidad del plasma decrece con la profundidad [26]. Para tener en cuenta este comportamiento, las áreas de los picos se normalizaron por el área total del espectro obtenido en cada pulso láser [32,33].

El análisis de cada pico del espectro LIPS se llevó a cabo utilizando Matlab ${ }^{\circledR}$ para obtener los perfiles de composición en profundidad. En todos los casos analizados se acumularon 6 pulsos láser en cada punto. El contenido en Fe y Ca para las tres muestras analizadas puede verse en la Figura 5 en función del número de pulsos. En los tres casos analizados el contenido en Fe es prácticamente constante durante los 40-60 
TABLA I. CONCENTRACIÓN ELEMENTAL (\% EN PESO) Y LAS CORRESPONDIENTES DESVIACIONES TÍPICAS (s) OBTENIDAS POR EDX EN, AL MENOS, 5 ÁREAS DEL ENGOBE Y LA PASTA DE LAS MUESTRAS DE TRICIO, ANDUJAR Y LA GRAUFESENQUE. SE INDICAN MEDIANTE LOS VALORES INFERIORES AL LÍMITE DE DETECCIÓN. SE HAN SEÑALADO CON * AQUELLOS ELEMENTOS QUE TAMBIÉN FUERON DETECTADOS POR LIPS EN LA VENTANA ESPECTRAL DE $260 \mathrm{~A} 340 \mathrm{~nm}$.

\begin{tabular}{|c|c|c|c|c|c|c|c|c|c|c|}
\hline Muestra & & & $\mathrm{O}$ & $\mathrm{Mg}^{*}$ & $\mathrm{Al}^{*}$ & $\mathrm{Si}^{*}$ & K & $\mathrm{Ca}^{*}$ & $\mathrm{Ti}^{*}$ & $\mathrm{Fe}^{*}$ \\
\hline \multirow{4}{*}{ Tricio } & \multirow{2}{*}{ Engobe } & media & 18,6 & - & 15,0 & 24,7 & 11,6 & 2,0 & - & 28,2 \\
\hline & & $\mathrm{s}$ & 7,2 & - & 3,5 & 7,1 & 2,5 & 0,6 & - & 11,4 \\
\hline & \multirow{2}{*}{ Pasta } & media & 35,9 & 0,4 & 6,6 & 31,4 & 4,4 & 3,7 & 0,8 & 16,8 \\
\hline & & $\mathrm{s}$ & 4,7 & 0,8 & 1,0 & 5,4 & 0,6 & 1,0 & 1,6 & 7,5 \\
\hline \multirow{4}{*}{ Andújar } & \multirow{2}{*}{ Engobe } & media & 28,5 & - & 17,0 & 30,4 & 8,7 & 5,2 & - & 14,2 \\
\hline & & $\mathrm{s}$ & 11,1 & - & 1,8 & 2,5 & 1,9 & 4,7 & - & 7,3 \\
\hline & \multirow{2}{*}{ Pasta } & media & 42,6 & 1,2 & 8,9 & 26,6 & 2,8 & 9,3 & 0,3 & 7,4 \\
\hline & & $\mathrm{s}$ & 3,2 & 0,8 & 1,0 & 4,9 & 1,6 & 2,4 & 0,5 & 2,0 \\
\hline \multirow{4}{*}{$\begin{array}{c}\text { La } \\
\text { Graufesenque }\end{array}$} & \multirow{2}{*}{ Engobe } & media & 39,4 & 0,6 & 12,6 & 33,5 & 5,8 & 1,7 & 0,3 & 6,2 \\
\hline & & $\mathrm{s}$ & 4,2 & 0,4 & 1,9 & 2,9 & 0,7 & 0,3 & 0,4 & 1,2 \\
\hline & \multirow{2}{*}{ Pasta } & media & 36,6 & 1,2 & 12,5 & 31,9 & 3,5 & 7,6 & 0,8 & 5,9 \\
\hline & & $\mathrm{s}$ & 4,1 & 0,4 & 0,4 & 1,4 & 0,1 & 1,5 & 0,3 & 0,9 \\
\hline
\end{tabular}
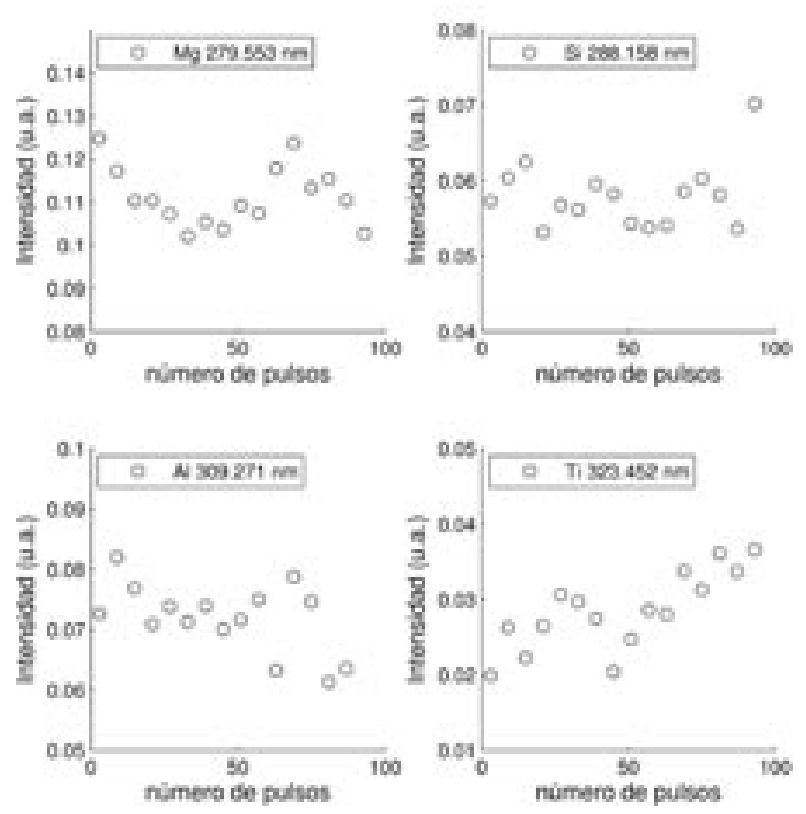

Fig. 6- Perfiles en profundidad de Mg(II) $279.553 \mathrm{~nm}, \mathrm{Si}$ (I) $288.158 \mathrm{~nm}$, $\mathrm{Al}$ (I) $309.271 \mathrm{~nm}$ y Ti(II) $323.452 \mathrm{~nm}$ en la muestra de La Graufesenque. Los valores de intensidad se han obtenido acumulando 6 pulsos láser.

primeros pulsos, correspondientes al engobe de la pieza, para sufrir una transición clara hasta valores más bajos en la zona correspondiente a la pasta de la cerámica. El comportamiento del Ca es el inverso al del Fe y la transición de valores bajos a un valor más alto se produce al mismo tiempo que la del Fe.

Esta transición tan clara observada para el Fe y Ca no se evidencia en los demás elementos, como puede apreciarse en la Figura 6, donde se representan las áreas de los picos $\mathrm{Mg}$, $\mathrm{Si} \mathrm{Al} \mathrm{y} \mathrm{Ti} \mathrm{en} \mathrm{la} \mathrm{muestra} \mathrm{de} \mathrm{La} \mathrm{Graufesenque.} \mathrm{El} \mathrm{contenido}$ en $\mathrm{Mg}$, Si y Ti experimenta fluctuaciones, pero se mantiene en torno a un valor constante; en cuanto al $\mathrm{Al}$ muestra una cierta tendencia a disminuir en la pasta, aunque no está muy clara. En las piezas de Tricio y Andújar el contenido en $\mathrm{Mg}$ y Ti no presenta variación apreciable del engobe a la pasta, mientras que el Si muestra un ligero incremento en la pasta de Andújar. El comportamiento del $\mathrm{Al}$ es distinto en cada una de las muestras analizadas. Por tanto, los perfiles de composición en profundidad en las tres piezas analizadas muestran que Fe y Ca son los elementos que mejor definen la transición entre engobe y pasta en los análisis LIPS de Terra Sigillata mientras que los gradientes de concentración de otros elementos no siguen una pauta definida. Además, los resultados están en buen acuerdo con aquellos obtenidos por EDX que se mostraron en la Tabla 1 e indican que en la elaboración del engobe se utilizaron arcillas enriquecidas en Fe y con un contenido en Ca menor que el de las pastas, como han observado otros autores [4-9].

\section{CONCLUSIONES}

Los resultados presentados en este trabajo muestran la capacidad de la técnica LIPS para obtener perfiles de composición en profundidad en restos de cerámica Terra Sigillata, permitiendo con ello distinguir diferencias en composición elemental entre engobe y pasta y caracterizar la transición entre ambas partes de la muestra. En todos los casos analizados el Ca y Fe son los elementos que mejor definen esta transición. Los perfiles de composición de otros elementos tales como $\mathrm{Si}$ o $\mathrm{Al}$ muestran un comportamiento diferente en cada una de las piezas analizadas. LIPS ha permitido además detectar en todas las muestras elementos tales como $\mathrm{Mg}$ y Ti que no fueron detectados por EDX. Estos resultados preliminares muestran que la técnica LIPS, en combinación con otras técnicas analíticas, puede proporcionar valiosa información en estudios arqueológicos sobre la cerámica Terra Sigillata, con la ventaja de tratarse de una técnica casi no destructiva, no requerir preparación de la muestra y permitir una gran rapidez en el análisis de un número importante de piezas. Para obtener resultados concluyentes en cuanto a las tecnologías de producción o en la asignación de procedencias de estas cerámicas mediante LIPS se hace necesario llevar a cabo el análisis de un mayor número de piezas.

\section{AGRADECIMIENTOS}

Los autores desean mostrar su agradecimiento a la Dra. M.C. López Pérez y al Museo de Prehistoria e Arqueoloxía de Vilalba, especialmente a su director Dr. J. Ramil Soneira por suministrarnos las piezas de Terra Sigillata y por la información y las facilidades prestadas. Este trabajo fue parcialmente subvencionado por Palladium Estudio de Arqueología S.L. y 
la Xunta de Galicia a través del Proyecto de I+D de Innovación Tecnológica de Empresas PGIDIT03CCP04E.

\section{BIBLIOGRAFÍA}

1. M.C. López Pérez. «Aportaciones al estudio del comercio romano a través de la cerámica de importación: La Terra Sigillata de la provincia de A Coruña». Tesis, Universidade de Santiago de Compostela, 2001.

2. M.S. Tite. "Archaeometry-an overview», pp. 347-356 en Physics Methods in Archaeometry. Proceedings of the International School of Physics Enrico Fermi, Course CLIV, Varenna on Lake Como (Italia), Junio 2003. Ed. M. Martini, M. Milazzo, M. Piacentini, Italian Physical Society. IOS Press, Amsterdam (The Netherlands) 2004.

3. M. Maggettti. «Chemical analyses of ancient ceramics: What for?». Chimia 55 923-930 (2001)

4. M.S. Tite, M.Bimson, I.C. Freestone. «An examination of the high gloss surface finishes of Greek Attic and Roman Samian wares». Archaeometry 24 117-126 (1982).

5. P. Mirti, A. Casoli, L. Calzetti. «Technology of production of fine pottery excavated on a western greek site investigated by scanning electron microscopy coupled with energy-dispersive X-ray detection». X-Ray Spectrom. 25 103-109 (1996).

6. A. Menéndez Llorente. «Caracterización química y mineralógica de un grupo de sigillatas de la comarca de Valdeorras (Orense)». Minius V 77-95 (1996).

7. P. Mirti, L. Appolonia, A. Cassoli. «Technological features of roman Terra Sigillata from galic and italian centres of production». J. Archaeol. Sci. 26 1427-1435 (1999).

8. P. Mirti. «X-ray microanalysis discloses the secrets of ancient greek and roman potters». X-Ray Spectrom. 29 63-72 (2000).

9. A. Zanco, G. Galetti. «Terra Sigillata imitations from Nyon (SW Switzerland): one of Fronto's workshops?». J. Cult. Heritage 2 109-116 (2001)

10. A.J. Polvorinos del Rio, V. Flores, M.A. Tabales, M.J. Hernández. «Caracterización y tecnología de materiales cerámicos romanos de los ss. I a III d.c. procedentes del Hospital de las Cinco Llagas de Sevilla». Bol. Soc. Esp. Ceram. V. 42 93-99 (2003).

11. L. Vendier, Ph. Sciau, E. Dooryhee. «Étude par diffraction des rayons $X$ des vernis rouges des sigillées du sud de la Gaule. Les ateliers de la Graufesenque». J. Phys. IV 12 189-196 (2002).

12. J.R. Gancedo, M. García, J.F. Marco, J. Palacios. «Mössbauer spectroscopic and SEM Study of Campanian and Terra Sigillata pottery from Spain». Hyperfine Interactions 41 791-794 (1988)

13. J.R. Castillo, J.M. Mir, J. Pérez-Arantegui, J. Tejada, J.R. Alabart. «Study of the provenance of Terra Sigillata by Mössbauer spectroscopy». Fresenius J. Anal. Chem. 341 611-614 (1991).

14. C. Lofrumento, A. Zoppi, E.M. Castellucci. «Micro Raman spectroscopy of ancient ceramics: a study of French sigillata wares». J. Raman Spectrosc. 35 650-655 (2004)

15. N.W. Bower, R.H. Bromund, R.H. Smith. «Atomic absortion for the archaeologist: An application to pottery from Pella of the Decapolis». J. Field Archaeol. 2 389-398 (1975).

16. P.J. Ballié, W.B. Stern. «Non-destructive surface analysis of roman Terra Sigillata: A possible tool in provenence studies?». Archaeometry 26 62-68 (1984).

17. J. Buxeda i Garrigós. «Alteration and contamination of archaeological ceramics: the perturbation problem». J. Archaeol. Sci. 26 295-313 (1999).

18. J. Poblome, P. Degryse, W. Viaene, R. Ottenburgs, M. Waelkens, R. Degeest, J. Naud. «The concept of a pottery production centre. An archaeometrical contribution from ancient Sagalassos». J. Archaeol. Sci. 29 873-882 (2002).

19. P. Mirti, R. Aruga, L. Appolonia, A. Casoli, M. Oddone. «On the role of major, minor and trace elements in provenancing ceramic material. A case study: Roman terra sigillata from Augusta Praetoria». Fresenius J. Anal. Chem. 348 396-401 (1994).

20. K.W. Slane, J.M. Elam, M.D. Glascock, H. Neff. «Compositional analysis of eastern sigillata A and related wares from Tel Anafa (Israel)». J. Archaeol. Sci. 21 51-64 (1994).

21.D. Anglos. «Laser-induced breakdown spectroscopy in art and archaeology». Appl. Spectrosc. 55 186A-205A (2001).

22. K. Melessanaki, M. Mateo, S.C. Ferrence, P.P. Betancourt, D. Anglos. «The application of LIBS for the analysis of archaeological ceramic and metal artifacts». Appl. Surf. Sci. 197-198 156-163 (2002).

23. F. Colao, R. Fantoi, V. Lazic, V. Spizzichino. «Laser-induced breakdown spectroscopy for semi-cuantitative and quantitative analyses of artworksapplication on multi-layered ceramics and copper based alloys». Spectrochim. Acta Part B 57 1219-1234 (2002).

24. J.M. Anzano, M.A. Villoria, I.B. Gornushkin, B.W. Smith. «Laser-induced plasma spectroscopy for characterization of archaeological material». Can. J. Anal. Sci. Spectrosc. 47 134-140 (2002).

25. Y. Yoon, T. Kim, M. Yang, K., Lee, G. Lee. «Quantitative analysis of pottery glaze by laser induced breakdown spectroscopy». Microchem. J. 68 251-256 (2001).

26. K. Muller, H. Stege. «Evaluation of the analytical potential of laser-induced breakdown spectrometry (LIBS) for the analysis of historical glasses». Archaeometry 45 421-433 (2003).

27. J.M. Vadillo, J.J. Laserna. «Laser-induced plasma spectrometry: truly a surface analytical tool». Spectrochim. Acta Part B 59 147-161 (2004).

28. L.J. Radziemski, D.A. Cremers. «Laser -induced plasmas and applications», pp.295-325 en: L.J. Radziemski, D.A. Cramers (Eds.) Capítulo 7, Marcel Dekker, New York, 1989,

29. M.P. Mateo, G.Nicolás, V. Piñon, J.C. Álvarez, A. Ramil, A. Yáñez. «Laser cleaning of Prestige tanker oil spill on coastal rocks controlled by spectrochemical analysis». Anal. Chim. Acta 524 27-32 (2004).

30. M.P. Mateo, G. Nicolás, V. Piñón, J.C. Álvarez, A. Ramil, A. Yáñez. «SALIPSA versatile software for semiautomatic analysis and processing of laserinduced plasma spectra». Spectrochim. Acta Part B, 2005 (In press).

31. NIST Spectroscopic Properties of Atoms and Atomic Ions Database, NIST Standard Reference Database 38.

32. D. Body, B.L. Chadwick. "Optimization of the spectral data processing in a LIBS simultaneous elemental analysis system». Spectrochim. Acta Part B 56 725-736 (2001).

33. A.J. López, G. Nicolás, M.P. Mateo, V. Piñon, M.J. Tobar, A. Ramil. "Compositional analysis of Hispanic Terra Sigillata by laser spectroscopy». Spectrochim. Acta Part B, 2005 (In press). 